\title{
Obstacle recognition from forward view images from trams
}

\author{
H. Miyayama, T. Ohya, T. Katori \& T. Izumi \\ Nihon University, Japan
}

\begin{abstract}
This research is concerned with the safe operation of tram systems.

We construct a warning system to the tram driver that recognizes obstacles from the forward view images from the tram cab. For this realization, front view images are processed, the obstacles in the image are recognized, and are judged either safe or dangerous.

This method's characteristic is to use a moving mono camera. At first, the danger area is limited by detecting the track. Static obstacles are detected by the variation of the intensity histogram between the tracks. Moving objects are detected by frame difference considering the tram's velocity in 3 dimensions. To recognize the obstacles the following characteristic parameters are adopted:

- Argument of diagonal in outside square of the detected obstacle

- Obstacle's width

By the recognition, obstacles in the images are classified into four categories: "Man", "Bicycle", "Car" and "The other". Collision or avoidance probability is judged by velocity vectors of the tram and obstacle. If the expected case of collision for "Man", "Bicycle" and "Car" is danger, then the tram driver is warned by the system.

We inspect the reliability of the image recognition in 13 scenes 14 images; the rate of correct judgment reaches $85 \%$ and collision judgment reaches $92 \%$.

Keywords: front view images of tram, frame difference considered tram's velocity, obstacle recognition, outside square, measurement used images, collision judgment.
\end{abstract}

\section{Introduction}

The aim of this study is to keep tram operation safe. 
Nowadays, the tram system is being re-evaluated all over the world. Good results are reported; in Japan, many existing routes are being extended and general railway lines are changing to tramways. The advanced tram vehicle is called the LRV(Light Rail Vehicle) in this trend [1].

The tram is convenient, but trams share road space with auto vehicles and pedestrians creating a weak point. Trams have to stop if any obstacles exist on the track, and this depends on the driver seeing the obstacle in time, an oversight causes a traffic accident.

Given this background, we present a safety support system for trams that automatically recognize some common obstacles. We devise a system that recognizes the obstacles from the image. The images are taken by a monocamera on the cab [2] of the track ahead. This system's aim is to support safe driving, the final responsibility is with the driver. Images are taken by a camera mounted in the cab, and the system warns the driver.

There is an automated vehicle operation in the ITS (Intelligent Transport System) technical result in the field [3]. Using image sensors, the system is able to automatically inspect invading obstacles on the track. If a traffic accident happens, it can be used to analysis the situation.

There are, however, some problems in using image processing technology:

- The camera is moving.

- The output of the process does not need detection, but recognition of obstacles, because some objects on the track are obstacles, but many are not. For instance, newspapers and so on.

- The outline of the obstacle is not constant, for example persons.

To address these problems, we moved the camera in the cab, and the process adopts three-dimensional coordinates and considers the velocity of the tram. To vary the outline of the obstacles, to recognize characteristic parameters, we adopt height and breadth measurements that are the sides of the obstacle's outside the square.

In this paper, we describe a method about recognition and collision judgment from front view images from the tram by image processing using a monocamera, track detection, and detection of static obstacles using an image brightness histogram. Detection of moving objects in three-dimensions coordinates the velocity of the tram, and recognition by width and argument of the outside square. Collision is judged by velocity vectors of the tram and obstacles. The judgment rate of correct obstacle recognition reaches $85 \%$, and collision judgment reaches $92 \%$.

\section{System composition}

Fig. 1 shows the composition of this system. For the system, front view images are taken.

A mono camera equips the cab. The images transmit to a PC through a video capture board; recognition and collision judgments are processed for the obstacles. The images are captured at $1 / 30$ [sec] time intervals. Currently this 


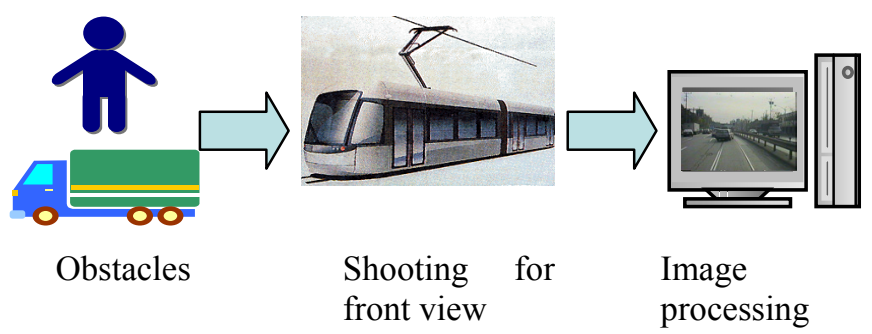

Figure 1: Composition of the system.

system drives offline, with images stored in HDD. But it will be possible to recognize obstacles in real time with the system, not to store, and to immediately transmit images to the system.

The image format is in bitmap form, the image size is $320 * 240$ pixels, quantitative steps are RGB 8[bit] (black: 0-white: 255), coordinates datum point in the image is upper left, and coordinates location are expressed as $(\mathrm{u}, \mathrm{v})$.

\section{Obstacles recognition from front view images of the tram}

\subsection{Procedure to recognize obstacles}

Fig.2 gives a block chart to recognize obstacles and judgement collision for tram's front view images.

First, the tracks in the images are detected to limit the danger area for input time sequence images. Static obstacles are detected by histogram variation between rails, and moving obstacles are detected by difference three-dimensional coordinates. To recognize obstacles, the width and argument of the outside square used as characteristic parameters. The obstacles are classified into 4 categories, "Man", "Bicycle", "Car", "The other". To judge collision vectors of the tram and obstacles are used.

\subsection{Detection of track}

As a first step, the tracks are detected, because the danger area is limited to between the rails. The detection processes to a frame picture. The edge is detected by a Sobel operator [4], noises are eliminated, thinning and line expansion are processed to the images, and a non gap continued line is extracted.

Sobel operators are given by (1). Fig.3 shows an example of track extraction.

$$
\left.\begin{array}{l}
f u=-f(u-1, v-1)+f(u+1, v-1)-2 f(u-1, v)+2 f(u+1, v)-f(u-1, v+1)+f(u+1, v+1) \\
f v=-f(u-1, v-1)-2 f(u, v-1)-f(u+1, v-1)+f(u-1, v+1)+2 f(u, v+1)+f(u+1, v+1)
\end{array}\right\}
$$

\subsection{Detection of static obstacles [5]}

Only road surface or tie and ballast exist between rails, if there is no obstacle on the track. In time sequence images, the histogram in that part of image does not vary quickly. If there are obstacles, the histogram varies. Therefore, static 


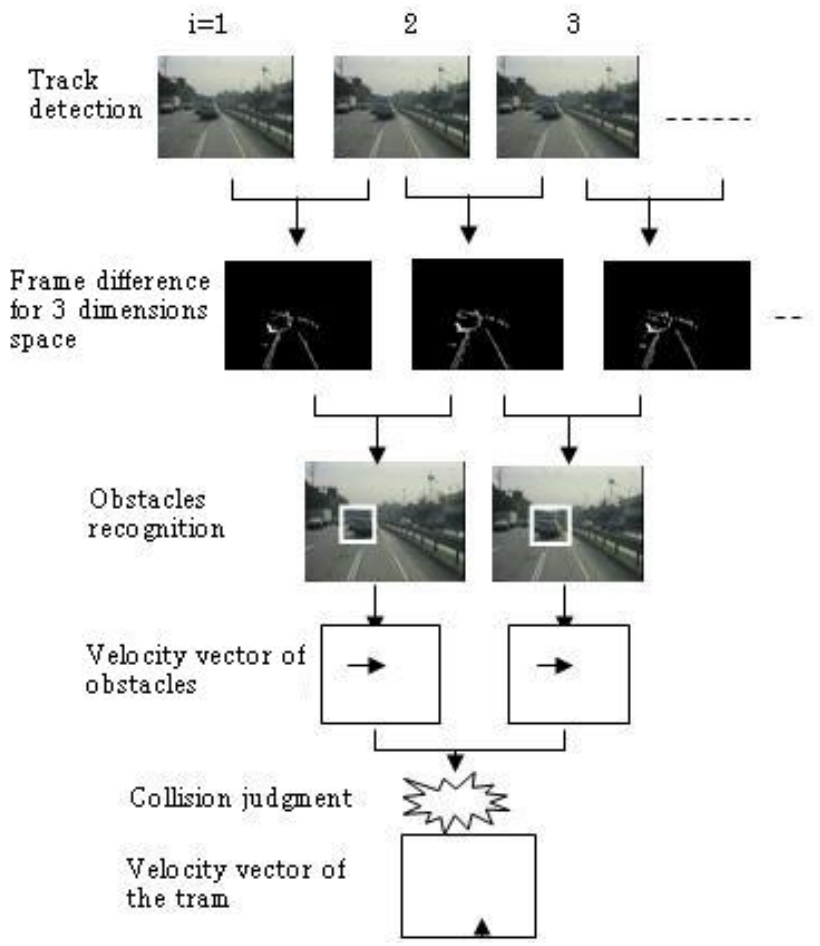

Figure 2: Block flow to recognize moving obstacles.

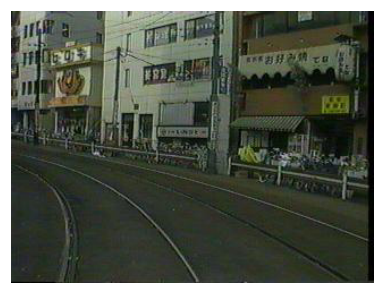

(a)

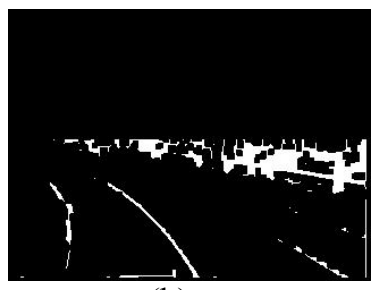

(b)

Figure 3: An example of track extraction. (a) An original image. (b) Extracted track.

obstacles are judged to exist, obstacles are detected by the histogram dispersion change, if the histogram dispersion $\sigma$ goes over threshold $\mathrm{th}_{1}$.

$$
t h_{1}<\left|\sigma_{i}-\sigma_{i-1}\right| \text {. }
$$

The histogram is varied for things such as paint on the road, but these are not original obstacles. These paints are excluded by Fourier transform, because these are geometric forms. 


\subsection{Detection of moving obstacles}

To extract moving objects from images, generally the extraction method is Background Subtraction or Frame difference. But it is impossible to use these methods, because the camera moves with the vehicle in the system. To detect moving obstacles, the camera's own velocity is gained by the speed indicator, and coordinates in 3 dimensions are obtained using Back-projection transfer; frame difference is processed in 3 dimensions.

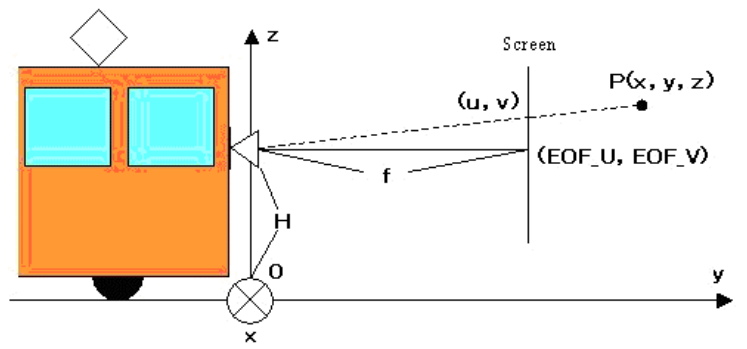

(a)

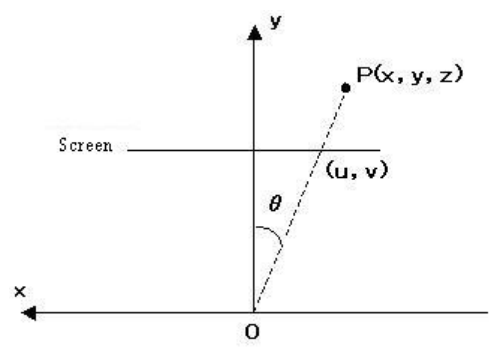

(b)

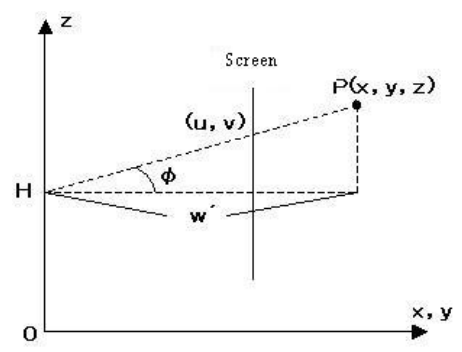

(c)

Figure 4: Back-projection frame of reference. (a) $x, y, z$ axis of coordinates, (b) $x-y$ plane, (c) x,y-z plane.

This system uses a mono-camera, but it is possible to calculate the 3 dimensional location using Back-projection transfer, because the gauge is constant, and obstacles touch ground exactly. The frame of reference transfers to $(\mathrm{x}, \mathrm{y}, \mathrm{z})=(\mathrm{x}, \mathrm{y}, 0)$ from $(\mathrm{u}, \mathrm{v})$.

Back-projection transfer is given by the following expression.

$$
\begin{gathered}
\theta=\tan ^{-1} \frac{u-E O F_{\_} U}{f} \ldots \\
\phi=\tan ^{-1} \frac{E O F_{\_} V-v}{\sqrt{f^{2}+\left(u-E O F_{-} U\right)^{2}}} \cdots \\
w^{\prime}=-\frac{H}{\tan \theta} \cdots \\
x=-w^{\prime} \sin \theta \cdots \\
y=w^{\prime} \cos \theta \cdots
\end{gathered}
$$


where

$\theta:$ Angle of depression

$\phi$ : Angle of elevation

EOF_U,EOF_V : vanishing point in image

$\mathrm{F}$ : Distance from camera to screen

$\mathrm{w}^{\prime}$ : Distance to object

$\mathrm{H}$ : Height of camera

The result of the only moving obstacle is detected in Fig.5.

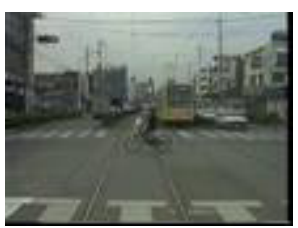

(a)

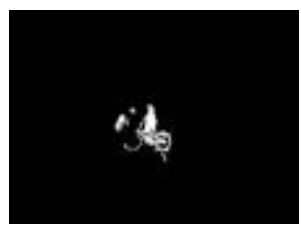

(b)

Figure 5: Result image of frame difference considered tram's velocity. (a) An original image. (b) The result image.

\subsection{Obstacle recognition}

It is necessary to warn the driver, if the forward obstacle is a man, bicycle or car. But if the obstacle is a newspaper or plastic bag, etc., it is not necessary. Therefore, obstacles are recognized in categories.

Characteristic parameters to recognize follow as

-Argument in Outside Square.

-Width of Outside Square.

Fig. 6 shows the characteristic parameters.

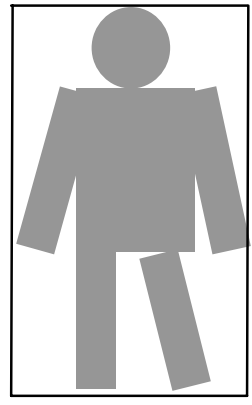

(a)

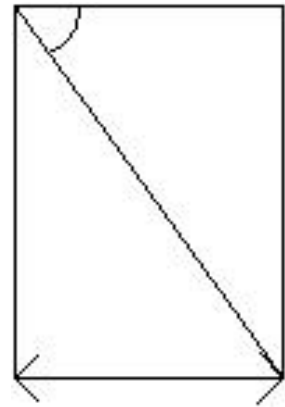

(b)

Figure 6: Characteristic parameters. (a) Outside square, (b) argument of diagonal and width. 


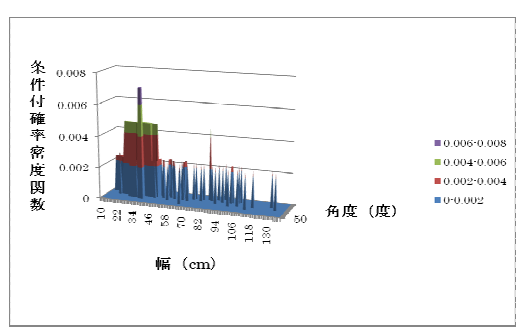

(a)

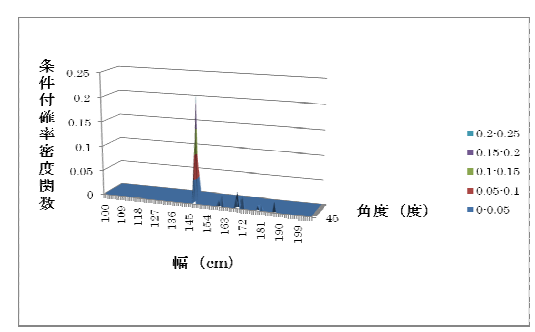

(b)

Figure 7: Examples of probability density function. (a) Man. (b) Car.

Obstacles are classified into 4 categories.

(1) Man

(2) Bicycle

(3) Car

(4) The other

(1) (3) are danger obstacles, (4) is not a danger one.

The conditional probability of 2 characteristic parameters are learned at each category; obstacles are recognized by Bayes' theorem [6], a popular statistical pattern recognition method. Fig.7 shows examples of the probability density function. Bayes' theorem is given by expression (7).

$$
\begin{aligned}
& P\left(\omega_{j} \mid x\right)=\frac{P\left(x \mid \omega_{j}\right) P\left(\omega_{j}\right)}{P(x)} \ldots \\
& P\left(\omega_{j}\right) \text { : Before probability } \\
& P\left(\omega_{j} \mid X\right) \text { : After probability on the basis of input pattern } X \\
& P(X) \text { : Probability of happen } X
\end{aligned}
$$

Conditional probability $\mathrm{P}\left(\omega_{\mathrm{j}} \mid \mathrm{X}\right)$ of each category is provided from Fig.7 for given $\mathrm{X}$. Obstacles belong to category $\mathrm{j}$ that has maximum conditional probability $\mathrm{P}_{\mathrm{j}}$.

\subsection{Collision judgment}

The system judges the collision probability for the tram and the obstacle, because there is a case of avoidance, if the result of the recognition is danger. In this study, we adopt the following conditions.

-LRV and obstacles run uniform acceleration.

-Obstacles move in straight motion.

The system traces detected obstacles in 3 dimensions, if the initial velocity of object $_{1}$ on $\left(\mathrm{x}_{1}, \mathrm{y}_{1}\right)$ is $\mathrm{v}_{10}$, and the acceleration is $\mathrm{a}_{10}$, if the initial velocity of object ${ }_{2}$ on $\left(\mathrm{x}_{2}, \mathrm{y}_{2}\right)$ is $\mathrm{v}_{20}$, and the acceleration is $\mathrm{a}_{20}$.

Velocity $v_{1}$ and $v_{2}$ at $t \mathrm{sec}$ later is

$$
\left.\begin{array}{l}
v_{1}=v_{10}+a_{10} t \\
v_{2}=v_{20}+a_{20} t
\end{array}\right\} .
$$


If object 1 and 2 arrive at collision point (c, c) at $t_{1}$ and $t_{2}$ later

$$
\left.\begin{array}{l}
t_{1}=\frac{c-y_{1}}{v_{10}+a_{10} t} \\
t_{2}=\frac{c-x_{2}}{v_{20}+a_{20} t}
\end{array}\right\} .
$$

because, if

$$
\left|t_{1}-t_{2}\right|<t h_{2} \cdots
$$

the system predicts a collision.

\section{Result and consideration}

This paper shows the results of obstacle recognition and collision judgments. Obstacle recognition uses real images, and collision judgment applied to model railway images, because it is difficult to take real collision scenes $(1 / 22.5$, obstacle is a figure in same scale). Fig. 8 shows the camera and the model railway used to take images, and Fig.9 shows an example of images with the model.
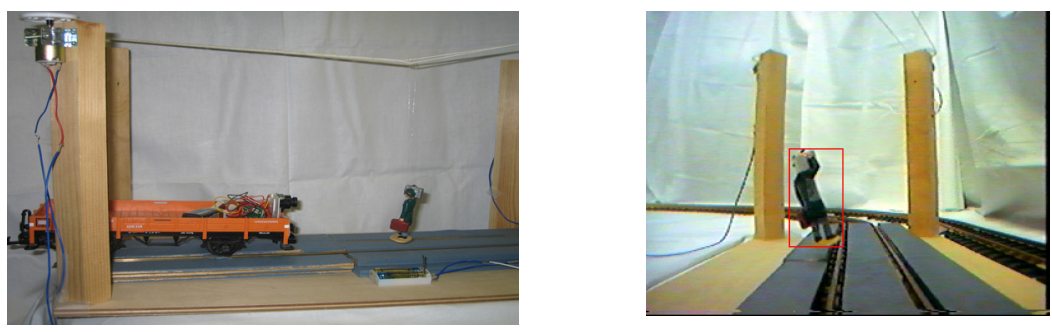

Figure 8: Camera and model Figure 9: An example of images. railway.

Table 1: $\quad$ Result of obstacles recognition.

\begin{tabular}{|l|l|l|l|l|}
\hline Input result & Man & Bicycle & Car & The others \\
\hline Man & 7 & 0 & 0 & 0 \\
\hline Bicycle & 1 & 5 & 0 & 0 \\
\hline Car & 3 & 0 & 11 & 0 \\
\hline The others & 1 & 0 & 0 & 0 \\
\hline
\end{tabular}

\subsection{Obstacle recognition}

Table 1 shows results of obstacle recognition. It shows recognition unknown patterns for 28 samples in 24 scenes using conditional probability function 
extracted from 150 trails. It is an excellent result, if there are diagonal answers only. In the table, Man is recognized exactly in such cases where the function is not distinguished. The category "The others" have various results. It is appropriate to judge "The other" if dismissed from "Man", "Bicycle" and "Car" by threshold. To improve the category of "The other", it is necessary to subdivide categories and to increase learning samples.

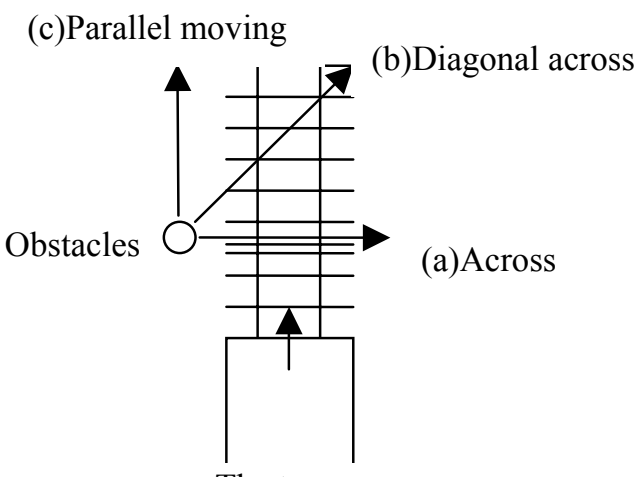

The tram

Figure 10: Condition for collision judgment.

The rate of recognition reaches $85 \%$. (Answer on the diagonal / all answers).

\subsection{Collision judgment}

Fig. 8 and table 2 show results of collision judgment. There are 13 scenes to judge. Table 3 shows the relation of conditions and results for collision judgment.

It gives good results for the most part, but there is a mis-judgement in the case of diagonal crossing of the track. It is important to calculate velocity, but camera oscillation makes a mistake in measuring velocity, which leads to a misjudgement. Although the number of scenes is small, the rate of collision judgment reaches $92 \%$.

Now, the system considers uniform acceleration and straight motion, but it is possible to improve the precision of collision judgment considering other motion patterns.

\section{Conclusion}

To enable safe operation of the tram vehicle, we have constructed a warning system for the tram driver, which recognizes and judges collision obstacles from the tram's front view image. Static obstacles are detected by variation of a histogram in the time sequential images between rails. 
Table 2: $\quad$ Condition for collision judgment.

\begin{tabular}{|l|c|c|}
\hline & Condition & Number of scene \\
\hline (a) & Across & 3 \\
\hline (b) & diagonal across & 8 \\
\hline (c) & parallel moving & 2 \\
\hline
\end{tabular}

Table 3: $\quad$ Result of collision judgment.

(a) Across

\begin{tabular}{|c|c|c|}
\hline Input judgment & collision & Avoidance \\
\hline collision & 2 & 0 \\
\hline avoidance & 0 & 1 \\
\hline
\end{tabular}

(b) Diagonal across

\begin{tabular}{|c|c|c|}
\hline Input judgment & collision & Avoidance \\
\hline collision & 3 & 1 \\
\hline avoidance & 0 & 4 \\
\hline
\end{tabular}

(c) Parallel moving

\begin{tabular}{|c|c|c|}
\hline Input judgment & collision & Avoidance \\
\hline collision & 0 & 0 \\
\hline avoidance & 0 & 3 \\
\hline
\end{tabular}

Moving obstacles are detected by the frame difference in 3 dimensional space. To recognize obstacles, characteristic parameters of the obstacle's argument and width of the outside square are looked at. Then the tram vehicle and obstacles are judged for collision or avoidance using vectors.

Judgment rate of correct recognition of obstacles reaches $85 \%$, and collision judgment reaches $92 \%$.

For future study:

- Detail classification for category "The other" in obstacle's recognition.

- Recognize static obstacles.

- Relation of recognition precision versus the distance between tram and obstacles.

- To apply or develop an algorithm for night scenes.

- Obstacle detection and recognition (by image data processing) from another view point such as Time-space images [7].

\section{Acknowledgement}

We thank Tomoaki Takahashi, a graduated student (now at The Japan electrical consulting Co. Ltd), who designed the component of the whole and difference disposal in 3 dimensional space and Yousuke Tomita (now at Oki Electric Industry Co. Ltd) who constructed static detection of obstacles.

\section{References}

[1] M.Yuhara, I.E.E. of Japan, Vol.119, 3, pp.148-151 (1999-3)(in Japanese) 
[2] T.Takahashi, T.Katori, Y.Takahashi and T.Izumi, "Pattern Recognition of Obstacles from Front View Images of LRV Using Frame Difference Considering Self-Vehicle Velocity”, I.E.E. of Japan, TER-04-17, pp.5156(2004-3) (in Japanese)

[3] "ITS road traffic sensing", Ohm-sya (2005-5)(in Japanese)

[4] M.Onoue et al, "Image Processing Handbook", Syokodo (1987) (in Japanese)

[5] Y.Tomita, H.Wada, T.Katori, Y.Takahashi and T.Izumi, "Static Obstacles Detection from Front View Images of LRV using Histogram and DFT", I.E.E. '05, 5-184(2005-3)(in Japanese)

[6] N.Funakubo, "Pattern recognition”, Kyoritsu-syuppan (1991)(in Japanese)

[7] T.Ohya, T.Katori, T.Izumi, "Obstacles detection from front view images of LRV used moving variance in Time-Space images", Proceeding of the 2008 IEICE general conference, D-11-67 (2008-3)(in Japanese) 